\title{
Inductively coupled probe for the measurement of partial discharges
}

\author{
Guillermo Robles, a) Juan Manuel Martínez, Mónica Rojas, and Javier Sanz \\ Department of Electrical Engineering, Universidad Carlos III, 28911 Madrid, Spain
}

(Received 4 February 2008; accepted 16 April 2008; published online 14 May 2008)

\begin{abstract}
Partial discharges are a transient phenomena whose measurement is remarkably important for electrical equipment diagnosis and maintenance. These discharges appear in the measurement circuit as very narrow current pulses of some nanoseconds. Therefore, discharge pulse detection is a particularly difficult problem, especially because they are superposed on the high voltage waveforms that cause them. In this paper, we present an inductively coupled probe able to measure this physical phenomenon by means of a very simple and inexpensive device that can be installed in the equipment under test. After modeling the probe, its response will be compared to that of commercial devices using both calibrated discharges and partial discharges occurring at real power. () 2008 American Institute of Physics. [DOI: 10.1063/1.2924207]
\end{abstract}

\section{INTRODUCTION}

The reliability of electrical equipment is an issue of profound interest nowadays because an unexpected failure may cause high economic costs in many industrial applications. The long-term behavior of power cables and electric machines is mainly determined by their insulation systems, whose integrity should be assured as much as possible.

Despite the fact that it is possible to measure the electric withstand capability from electrical equipment-electric breakdown strength-its aging is a cumbersome problem greatly affected by partial discharges (PDs). These are local electrical breakdown phenomena located at sites less capable of withstanding electric field or those subjected to an enhanced one. ${ }^{1}$ Partial discharges are a rapid flow of charge carriers lasting some nanoseconds that do not provoke a direct failure of the whole dielectric, but provide electrical aging by means of ionic bombardment and chemical attack. ${ }^{2}$ This is the reason why there is great interest in measuring this phenomenon, as it may help in predictive maintenance.

Currently, normalized measurements are made by means of capacitive dividers, whose bandwidth is limited to the $100-500 \mathrm{kHz}$ range ${ }^{3,4}$ They are able to measure PD magnitude and its phase occurrence referring to the power frequency, but their bandwidth limitations do not allow measurement of the waveforms of the partial discharge propagation. ${ }^{5}$

Ultrasonic and rf probes have been proposed to measure PD in electrical machines. Unfortunately, they require dismantling the machine and considerable operator skills to ensure that one coil is in good condition. ${ }^{6}$

There have been serious efforts to measure PD pulse waveforms since they seem to provide information regarding insulation diagnosis, ${ }^{7}$ but their detection is difficult in real industrial environments where these high frequency and low amplitude pulses, with frequency spectra ranging from $100 \mathrm{kHz}$ to $100 \mathrm{MHz}$, appear superposed to power frequency high voltages and currents. One solution is the use of high frequency current transformers (HFCTs) because they

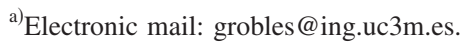

provide galvanic isolation and have high bandwidths capable of measuring PD pulse waveforms. However, they are based on expensive high frequency ferromagnetic materials that must show as low hysteresis as possible and, for some power ratings, they may saturate when connected to wires with high currents. These are the reasons to try to replace this device with an air-cored high frequency transformer, but as there is no ferrite core to concentrate the flux in, its sensitivity, up to now, is very low for detecting PDs. ${ }^{6}$

Another configuration to measure partial discharges is based on a self-integrating Rogowski coil. This probe uses a simple resistor to separate the poles of the frequency response and obtains a frequency window where the signal is integrated. ${ }^{8}$ The configuration has low sensitivity without amplifiers and the equivalent circuit requires a more complex model. ${ }^{9}$

This paper proposes a nonmagnetic core inductive device able to detect partial discharges at insulation systems in electric equipment. The probe location assures a better sensitivity than previous air-cored transformers while being a cheaper solution to detect fast PD transients. The theoretical approach to the probe response justifies its good behavior. The probe sensitivity, measurement reproducibility, and linear response will be demonstrated detecting known PD events. Finally, its capability to detect high frequency pulses when different ac high voltage levels are applied to an insulation system is tested.

\section{PROBE DESIGN AND OPERATING PRINCIPLE}

The total length of the loop plays an essential role on the design of the sensor. By assuming a maximum frequency value for the partial discharge pulse of $100 \mathrm{MHz}$, the maximum length of a conductor to be modeled by a lumped parameter equivalent circuit is about $0.3 \mathrm{~m}$, that is, one-tenth of the basic wavelength value that amounts to $3 \mathrm{~m}$. Under this constraint, there will not be reflections in the probe. The final prototype, printed on a circuit board, is a loop $12 \mathrm{~cm}$ long and $0.9 \mathrm{~cm}$ wide, Fig. 1, thus yielding a total length of $25.8 \mathrm{~cm}$ that fulfills the maximum length constraint. The wire conducting the partial discharge signal is attached to 


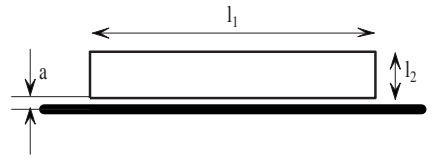

FIG. 1. Geometric parameters in the inductive sensor.

two connectors and a coaxial connector is used as the output of the probe. The sensor operates directly upon two Maxwell equations, usually termed as Ampère and Faraday laws. According to the first law, the current flowing through the main conductor creates a magnetic field of circular shape. As the partial discharge is a sudden charge transfer inside the dielectric $^{10}$ that appears as a very short duration current pulse in the measuring circuit, the variation of the corresponding magnetic field induces a voltage at the ends of the turn. This voltage is proportional to the derivative of the current in the main wire, Eq. (1), so it should be integrated to obtain the signal corresponding to the partial discharge. $M$ is the mutual inductance between the turn and the main conductor and $i$ is the primary current.

$$
e=M \frac{d i}{d t} \text {. }
$$

The shape of the loop has been chosen after a study of how the geometric parameters affect the mutual inductance. By assuming that the main wire is infinitely long, $M$ can be written as

$$
M=\frac{\mu_{0}}{2 \pi} l_{1} \log \frac{l_{2}+a}{a},
$$

where the parameters are shown in Fig. 1.

To maximize $M$ and hence, the sensitivity of the probe, $l_{1}$ and $l_{2}$ should be as long as possible and $a$ should be the smallest distance. In the design, $a$ is defined as the distance between the middle lines of the conductors and its value is $1.016 \mathrm{~mm}(0.04 \mathrm{in}$.). The widths of the conductor of the turn and the main conductor are $0.381 \mathrm{~mm}$ (0.015 in.) and $1.016 \mathrm{~mm}$ (0.04 in.) respectively. Finally, the dimensions of the rectangle are $l_{1}=12 \mathrm{~cm}$ and $l_{2}=0.9 \mathrm{~cm}$.

As the total length is limited to less than $30 \mathrm{~cm}$ and $l_{2}$ is inside a logarithm, $l_{1}$ is the parameter that most influences the mutual inductance. Finally, the corners of the rectangle were blunted to prevent undesired electromagnetic emissions.

\section{EQUIVALENT HIGH FREQUENCY CIRCUIT}

As mentioned before, in the prototype, great effort has been made to keep the distances small compared to the wavelength of the induced voltage. Therefore, the electric circuit can be considered with lumped parameters, as shown in Fig. 2. This schematic is a variation of the equivalent circuit in $T$ for a transmission line. The induced voltage is termed $e$ and $R$ and $L$ are the total resistance and inductance, whereas $C_{1}$ and $C_{2}$ are the total capacitances between the long sides and the short sides of the rectangle, respectively. By estimating these parameters and solving the transfer function between the output and input voltages would give the self-resonance frequency of the system. This frequency

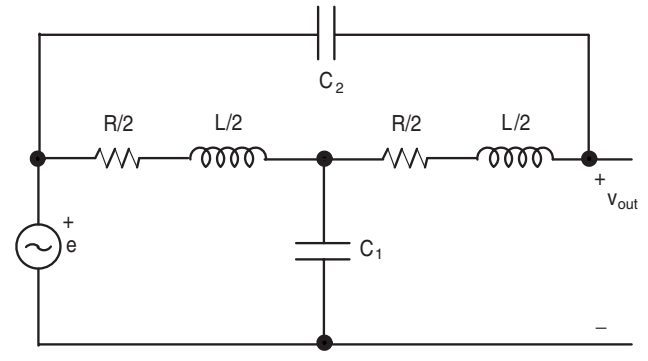

FIG. 2. Electric equivalent circuit.

should be much higher than the highest frequency of consideration in the spectrum of the partial discharge signal to avoid oscillations.

The electric parameters are calculated for a simplified loop such as that shown in Fig. 1. The width of the conductors is termed $h$ in the equations.

The resistance depends on frequency and it is modified by the skin depth in the conductor. Considering the shape of the conductor as a strip with thickness $d$ and width $h$, the general formula for the internal impedance is ${ }^{11}$

$Z_{i}=\frac{1+j}{2 h} R_{s} l \frac{\cos (d / 2 \delta) \cosh (d / 2 \delta)+j \sin (d / 2 \delta) \sinh (d / 2 \delta)}{\cos (d / 2 \delta) \sinh (d / 2 \delta)+j \sin (d / 2 \delta) \cosh (d / 2 \delta)}$

with

$$
R_{s}=\frac{1}{\delta \sigma}
$$

where $R_{s}$ is the surface resistance, $\delta$ is the skin depth, $\sigma$ is the conductivity of copper, $d=35 \mu \mathrm{m}$, and $l$ is the length of the conductor.

The resistance of the conductor is obtained from the real part of Eq. (3) and is shown in Eq. (5).

$$
R_{i}=\frac{l_{i}}{2 h} R_{s} \frac{e^{-2 d / \delta}+4 e^{-d / \delta} \cos (d / 2 \delta) \sin (d / 2 \delta)-1}{e^{-2 d / \delta}-2 e^{-d / \delta} \cos (d / \delta)+1} .
$$

With these considerations, two resistances in function of the skin depth, and hence, in function of the frequency, are obtained. They are defined as $R_{1}$ for the long sides with $l_{i}=2 l_{1}$, and $R_{2}$ for the short sides of the rectangle with $l_{i}=2 l_{2}$. In the schematic, $R=R_{1}+R_{2}$.

The capacitance between the long lines is calculated with Eq. (6) considering that $\epsilon_{r}=4.5$ is the relative permittivity of the dielectric FR-4, which is the base of the printed circuit board. ${ }^{12,13}$

$$
C_{1}=\frac{\pi \epsilon_{r} \epsilon_{0} l_{1}}{\ln \left(\frac{2 l_{2}}{h}\right)}=3.896 \mathrm{pF}
$$

Analogously, the capacitance between short lines is based on Eq. (7).

$$
C_{2}=\frac{\pi \epsilon_{r} \epsilon_{0} l_{2}}{\ln \left(\frac{2 l_{1}}{h}\right)}=0.1748 \mathrm{pF}
$$

With $C_{1} \gg C_{2}$, this last capacitance can be considered negligible, so $C=C_{1}$ for the following calculations. 

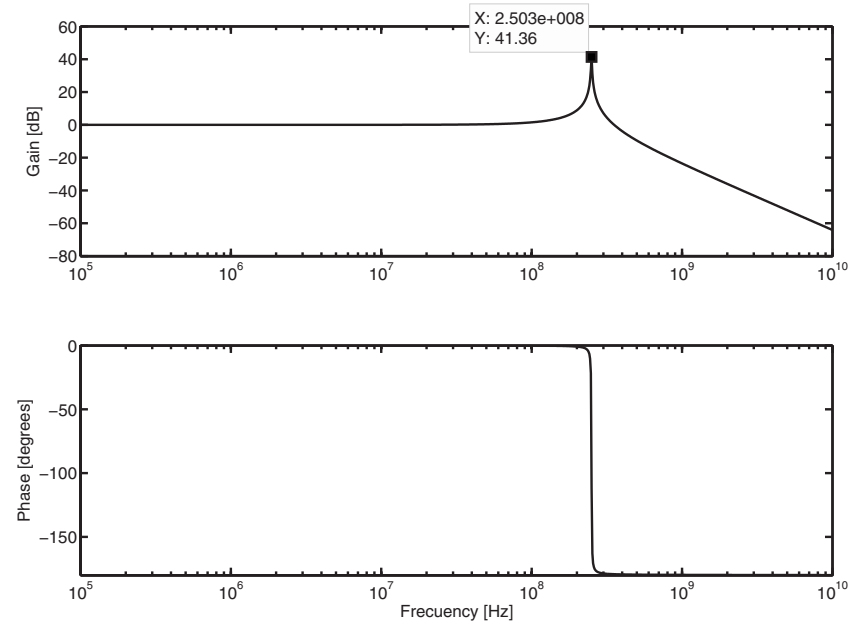

FIG. 3. Frequency response for the inductive probe with the dimensions given in Sec. II.

The inductance can be calculated considering the relation between the inductance and capacitance per unit length, $L_{l}$ and $C_{l},{ }^{12,13}$

$$
L_{l} C_{l}=\mu \epsilon,
$$

with $\mu=\mu_{0}$ for the dielectric FR-4. Hence,

$$
L_{1}=\frac{\mu l_{1}}{\pi} \ln \left(\frac{2 l_{2}}{h}\right)=185.06 \mathrm{nH}
$$

and

$$
L_{2}=\frac{\mu l_{2}}{\pi} \ln \left(\frac{2 l_{1}}{h}\right)=23.2 \mathrm{nH} .
$$

In Fig. 2, $L=L_{1}+L_{2}=208.26 \mathrm{nH}$.

Additionally, the internal inductance in the strip conductor might contribute to the total inductance. It can be calculated from the complex part of the impedance $Z_{i}$, Eq. (3). It depends on the frequency so, when this is increased, the inductance drops. In the frequency range of interest, the values are $2.49 \mathrm{nH}$ at $1 \mathrm{MHz}$ and $1.43 \mathrm{nH}$ at $100 \mathrm{MHz}$ which are negligible compared to inductance $L$ and definitely are not considered in the model.

The mutual inductance for one turn follows Eq. (2), rewritten below for the sake of clarity.

$$
M=\frac{\mu_{0}}{2 \pi} l_{1} \log \frac{l_{2}+a}{a}=54.9 \mathrm{nH} .
$$

Hence, in Fig. 2, $e=54.9 \times 10^{-9} d i / d t$. Although the gain given by the mutual inductance might seem dramatically low, signals with frequencies in the band of megahertz would give induced voltages of millivolts that are easily measured even without amplifiers.

As far as the transfer function between output voltage and induced voltage is concerned, solving the circuit in Fig. 2, yields

$$
\frac{V_{\text {out }}}{E}=\frac{2}{L C s^{2}+R C s+2},
$$

where $V_{\text {out }}$ is the Laplace transformation of $v_{\text {out }}(t)$ and $E$ is the transformation of the induced voltage. Bear in mind that
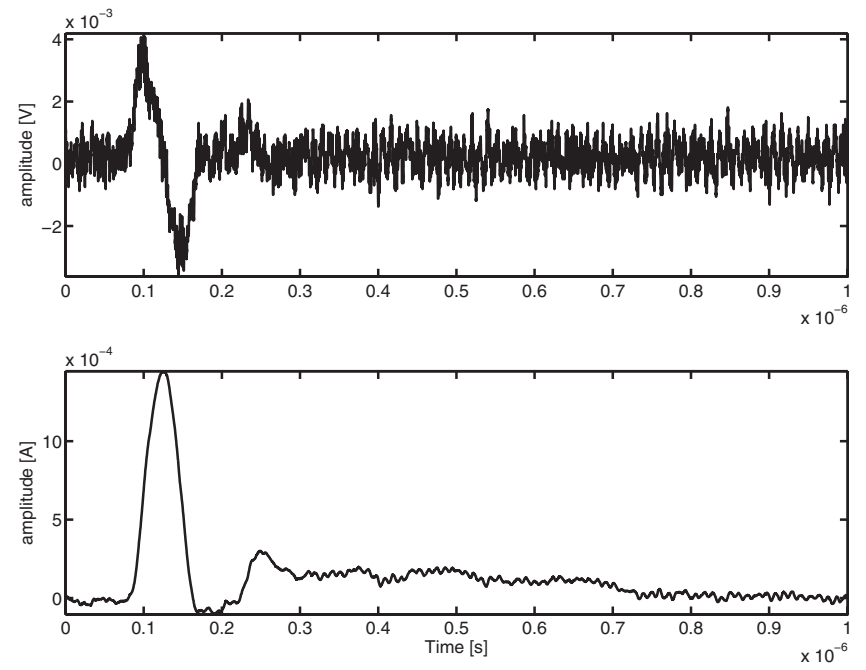

FIG. 4. Voltage signal obtained with the probe and the same signal integrated for a $100 \mathrm{pC}$ pulse.

from Eq. (5), the resistance $R$ depends on frequency and this is considered in the plot. Substituting the electric parameters in the function, the complex pole is found at $250 \mathrm{MHz}$, which is sufficiently far from the frequency content in a partial discharge, Fig. 3. At the frequencies of interest, the gain is $0 \mathrm{~dB}$ and the output voltage is directly the induced voltage in the loop.

\section{CALIBRATION}

To validate Eq. (11), a simple setup was designed. An Agilent 33120A signal generator was connected to a $110 \Omega$ resistor to limit the current, and to the main conductor of the prototype. The peak voltage was set to $2 \mathrm{~V}$, so the current was $18.2 \mathrm{~mA}$. The waveforms, ranging from 1 to $15 \mathrm{MHz}$, were sinusoidal so that the derivative could be easily found. The output of the sensor was connected to the $50 \Omega$ input of an oscilloscope and amplitudes were taken in the frequency sweep. Linearity in the relation between input and output was held in the frequency range with the peak of $v_{\text {out }}$ increasing $6.25 \mathrm{mV} / \mathrm{MHz}$. For sinusoidal waveforms, the amplitude of the output voltage is $V=M \omega I$, then,

$$
M=\frac{V}{\omega I}=\frac{6.25 \times 10^{-3}}{2 \pi 10^{6} \times 18.2 \times 10^{-3}}=54.65 \mathrm{nH} .
$$

This result guarantees that the mutual inductance obtained with Eq. (2) is valid and allows us to calculate the current through the main conductor by simply integrating the voltage and dividing it into $M$.

In order to measure charge transfer-in $\mathrm{pC}$ - through the dielectric, a calibration procedure is needed previous to any high voltage measurement, ${ }^{6}$ to correlate the magnitude of the transferred electrical charge inside the insulation with the voltage signal detected by the sensor in the external circuit. This is accomplished by using a standardized PD calibrator connected in parallel to the equipment under test that injects a charge of known magnitude by means of a low signal voltage pulse in absence of applied voltage, Fig. 6. Two different levels of apparent injected charge have been used in this 

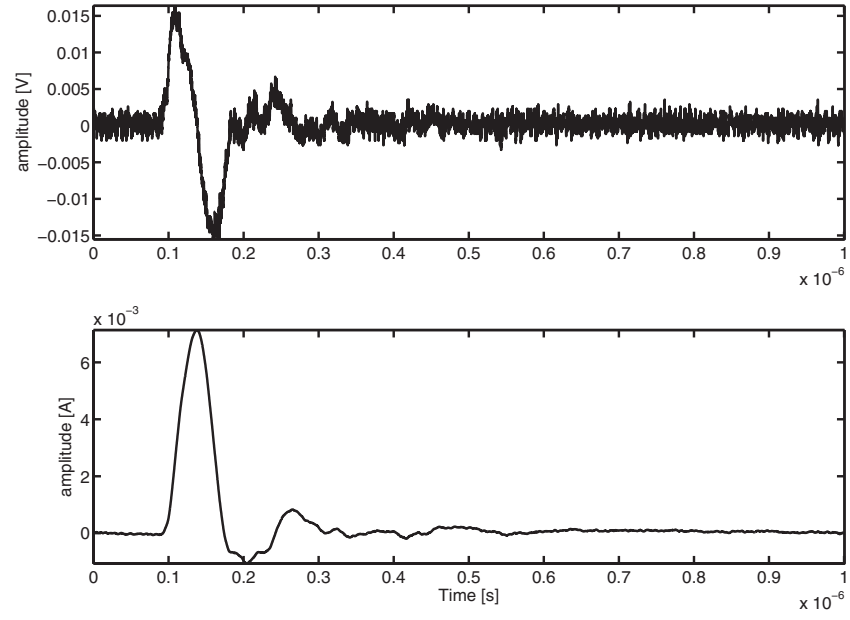

FIG. 5. Voltage signal obtained with the probe and the same signal integrated for a $500 \mathrm{pC}$ pulse.

calibration to both establish the minimum sensitivity or detection threshold of the probe, and to compare both signals detected with the probe.

The first plot in Fig. 4 shows the signal obtained with the sensor for a pulse of $100 \mathrm{pC}$. The second plot is the signal integrated and divided into $M$. Figure 5 is the same for a calibration pulse of $500 \mathrm{pC}$. The peaks of these currents are 1.446 and $7.135 \mathrm{~mA}$ for 100 and $500 \mathrm{pC}$, respectively. This corroborates the fact that one is five times greater than the other. Notice that the sensor output is directly connected to the oscilloscope and the use of an amplifier is not necessary. The integration is made offline with MATLAB using the raw data from the oscilloscope. Integration behaves as a low pass filter so, when the output signal is processed, noise is reduced. In the whole acquisition setup, no additional filter is required.

\section{PULSE MEASUREMENT}

Partial discharges are measured with the setup shown in Fig. 6. A high voltage transformer capable of reaching peaks of $100 \mathrm{kV}$ is connected to a planar electrode. A dielectric provides insulation between the electrode and ground. The inductive sensor and a commercial high frequency current transformer are placed in the wire to ground. The outputs of the two probes are connected to a Tektronix TDS 5032B

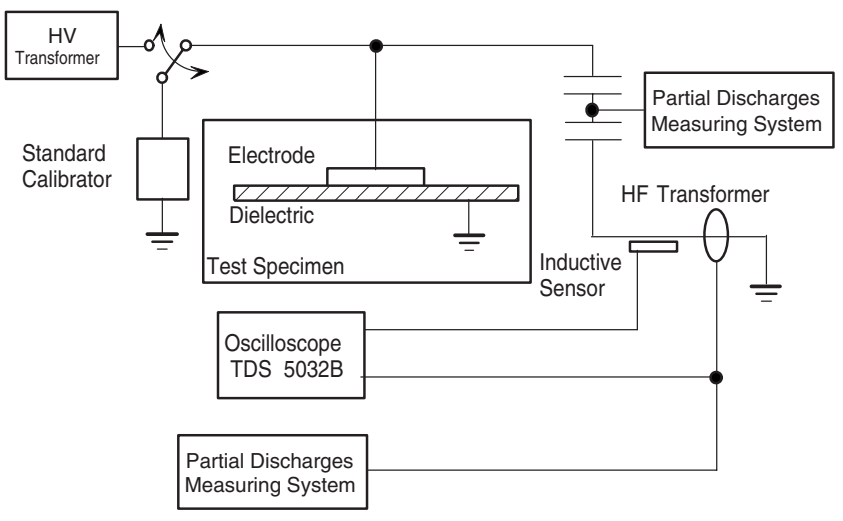

FIG. 6. Experimental setup for partial discharge testing.
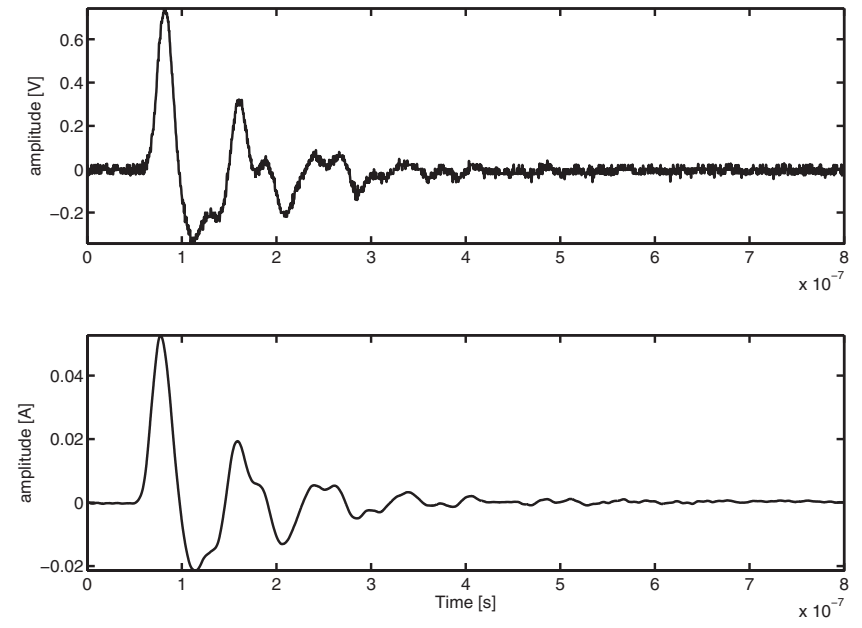

FIG. 7. Current pulse corresponding to a partial discharge at $1700 \mathrm{~V}$ measured with a high frequency transformer (above) and the same signal in amperes captured with the sensor and integrated (below).

oscilloscope with coaxial cables of the same lengths and the output of the HFCT transformer is also connected to a commercial partial discharge measuring system. Additionally, the high voltage transformer has a capacitive divider to connect another traditional partial discharge measuring system with characteristics similar to the first one. These two systems are connected to compare measurements and, hence, guarantee that partial discharges are effectively happening and their waveform and nature are known.

Figures 7 and 8 show two partial discharge pulses produced on the test specimen at 1700 and $2400 \mathrm{~V}$, respectively. The upper plot is the signal measured with the high frequency transformer and the lower plot is the signal obtained with the probe, integrated and divided into the mutual inductance. It can also be appreciated how higher voltages lead to higher amplitudes in the partial discharges. These figures demonstrate that the inductive sensor measures this transient phenomenon accurately.
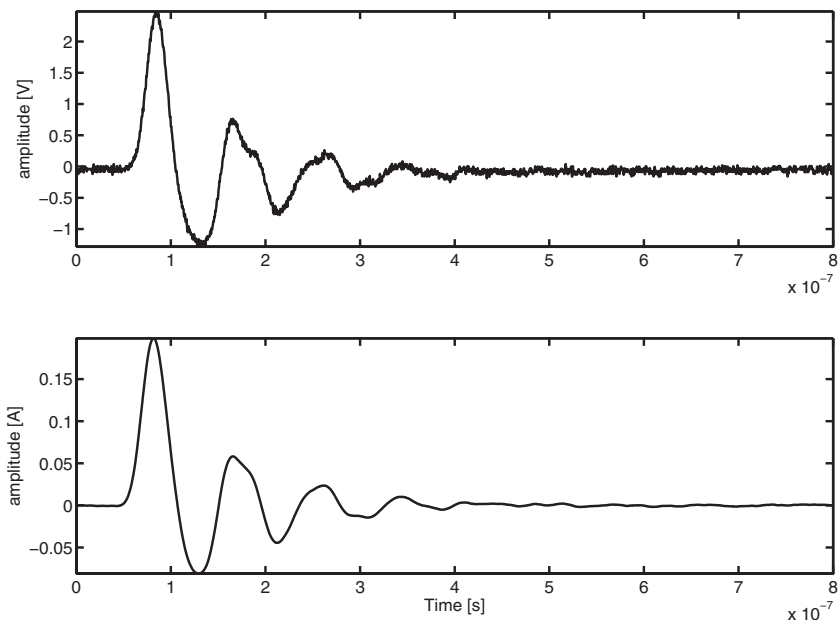

FIG. 8. Current pulse corresponding to a partial discharge at $2400 \mathrm{~V}$ measured with a high frequency transformer (above) and the same signal in amperes captured with the sensor and integrated (below). 


\section{DISCUSSION}

A partial discharge measuring sensor was designed, constructed, calibrated, and tested. The device is based on an inductively coupled probe and is able to detect partial discharge pulse waveforms accurately. The device works inside the appropriate bandwidth and represents a simple and cheap alternative for detecting these high frequency pulses. Since it measures the pulse derivative, an offline signal integration has been done. Future works will be aimed at comparing different approaches to obtain the original PD waveform integrating the signal given by the sensor and at exploring the possibility of maximizing the sensitivity by modifying the geometry.

\section{ACKNOWLEDGMENTS}

This research has been supported by the Spanish Science and Technology Ministry under Contract No. DPI 200615625-C03-02. Tests have been made in the High Voltage
Research and Tests Laboratory of Universidad Carlos III de Madrid (LINEALT).

${ }^{1}$ V. S. Filho and R. Robert, Rev. Sci. Instrum. 73, 1288 (2002).

${ }^{2}$ L. Dissado and J. Fotherhill, Electrical Degradation and Breakdown in Polymers, Materials and Devices, (IEE, London, 1992).

3 "High voltage test techniques_-Partial discharge measurements," Document No. IEC 60270, 2000.

4 "IEEE trial use guide to the measurement of partial discharges in rotating machinery," IEEE Standard No. 1434-2000, 2000.

${ }^{5}$ R. Bartnikas and E. J. McMahon, Engineering Dielectrics, Corona Measurements and Interpretation, Vol. 1 (ASTM, Philadelphia, 1979).

${ }^{6}$ G. Stone, E. Boutler, I. Culbert, and H. Dhirani, Electrical Insulation for Rotating Machines: Design, Evaluation, Aging, Testing and Repair, Series on Power Engineering (IEEE, Piscataway, NJ, 2004).

${ }^{7}$ A. Cavallini, G. Montanari, A. Contin, and F. Pulletti, IEEE Electr. Insul. Mag. (USA) 19, 22 (2003).

${ }^{8}$ M. Argueso, G. Robles, and J. Sanz, Rev. Sci. Instrum. 76, 065107 (2005).

${ }^{9}$ V. Dubickas and H. Edin, IEEE Trans. Instrum. Meas. 56, 2284 (2007).

${ }^{10}$ R. J. V. Brunt and S. Kulkarni, Rev. Sci. Instrum. 60, 3012 (1989).

${ }^{11}$ B. D. Popovic, Introductory Engineering Electromagnetics (AddisonWesley, Reading, MA, 1971).

${ }^{12}$ W. Stutzman and G. Thiele, Antenna Theory and Design (Wiley, New York, 1998).

${ }^{13}$ W. Hayt, Engineering Electromagnetics (McGraw Hill, New York, 2006). 